\title{
Effect of collective neutrino flavor oscillations on $\nu$ p-process nucleosynthesis
}

\author{
G. Martínez-Pinedo ${ }^{1, a}$, B. Ziebarth ${ }^{1}$, T. Fischer ${ }^{1,2}$, and K. Langanke ${ }^{1,2,3}$ \\ 1 GSI Helmholtzzentrum für Schwerionenforschung, Planckstraße 1, 64291 Darmstadt, Germany \\ 2 Technische Universität Darmstadt, Schlossgartenstraße 9, 64289 Darmstadt, Germany \\ 3 Frankfurt Institute for Advanced Studies, Ruth-Moufang Straße 1, Frankfurt, Germany
}

Received: 9 August 2011

Published online: 25 August 2011

(c) The Author(s) 2011. This article is published with open access at Springerlink.com Communicated by U.-G. Meißner

\begin{abstract}
The $\nu p$ process is a primary nucleosynthesis process which occurs in core-collapse supernovae. An essential role in this process is being played by electron antineutrinos. They generate, by absorption on protons, a supply of neutrons which, by $(n, p)$ reactions, allow to overcome waiting point nuclei with rather long beta-decay and proton-capture lifetimes. The synthesis of heavy elements by the $\nu p$ process depends sensitively on the $\bar{\nu}_{e}$ luminosity and spectrum. As has been shown recently, the latter are affected by collective neutrino flavor oscillations which can swap the $\bar{\nu}_{e}$ and $\bar{\nu}_{\mu, \tau}$ spectra above a certain split energy. Assuming such a swap scenario, we have studied the impact of collective neutrino flavor oscillations on the $\nu p$-process nucleosynthesis. Our results show that the production of light $p$-nuclei up to mass number $A=108$ is very sensitive to collective neutrino oscillations.
\end{abstract}

Core-collapse supernova explosions have been identified as the site for explosive nucleosynthesis [1]. Of particular interest, here, is the matter that is ejected from the surface of the newly born neutron star due to latetime neutrino heating, known as the neutrino-driven wind. It develops on time scales of seconds after the onset of the explosion. Due to the high temperatures at the surface, this matter is ejected as free protons and neutrons. Upon reaching cooler regions with increasing distance from the neutron star, nucleons can be combined into nuclei. The outcome of this nucleosynthesis depends on the proton-to-baryon ratio of the ejected matter. This ratio is determined by the competition of electron neutrino and antineutrino absorption on nucleons and their inverse reactions

$$
\nu_{e}+n \rightleftarrows p+e^{-}, \quad \bar{\nu}_{e}+p \rightleftarrows n+e^{+} .
$$

It strongly depends on the neutrino and antineutrino luminosities and spectra. Simulations $[2-5]$ indicate that in an early phase of the explosion, the ejected matter is proton-rich; i.e. the proton-to-neutron ratio $Y_{e}$ is larger than 0.5. When this proton-rich matter expands and cools, nuclei can form resulting in a composition dominated by $N=Z$ nuclei, mainly ${ }^{56} \mathrm{Ni}$ and ${ }^{4} \mathrm{He}$, and protons [6].

\footnotetext{
a e-mail: g.martinez@gsi.de
}

Without the further inclusion of neutrino and antineutrino reactions the composition of this matter will finally consist of protons, alpha-particles, and heavy (Fe-group) nuclei (in nucleosynthesis terms a proton- and alpha-rich freeze-out), with enhanced abundances of ${ }^{45} \mathrm{Sc},{ }^{49} \mathrm{Ti}$, and ${ }^{64} \mathrm{Zn}[7,8]$, solving the longstanding puzzle of underproduction of these elements in stellar nucleosynthesis studies. The matter flow stops at $A=64$, due to the long betadecay half-live of ${ }^{64} \mathrm{Ge}, 63.7(25) \mathrm{s}$, which is much longer than the expansion time scale, and the small proton separation energy of ${ }^{65} \mathrm{As},-90(85) \mathrm{keV}$ [9], which suppresses proton capture on ${ }^{64} \mathrm{Ge}$ at the high temperatures involved.

However, neutrino reactions play an essential role during the nucleosynthesis. $N \sim Z$ nuclei are practically inert to neutrino captures (which convert a neutron into a proton), because such reactions are endoergic for neutrondeficient nuclei located away from the valley of stability. The situation is different for electron antineutrinos that are captured in a typical time of a few seconds, both on protons and nuclei, at distances of $\sim 500 \mathrm{~km}$. This time scale is much shorter than the beta-decay half-lives of the most abundant heavy nuclei reached without neutrino interactions (e.g., $\left.{ }^{56} \mathrm{Ni},{ }^{64} \mathrm{Ge}\right)$. As protons are more abundant than heavy nuclei, electron antineutrino capture occurs predominantly on protons, causing a residual density of free neutrons of $10^{14}-10^{15} \mathrm{~cm}^{-3}$ for several seconds, when the temperatures are in the range of $1-3 \times 10^{9} \mathrm{~K}$. 


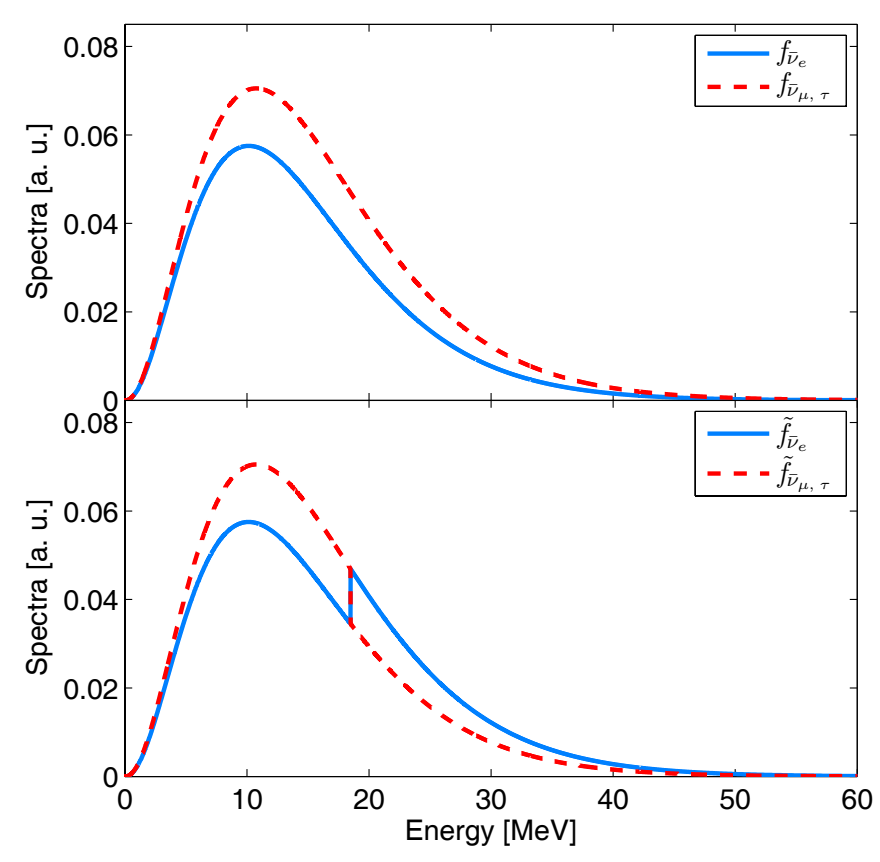

Fig. 1. Upper panel: $\bar{\nu}_{e}$ and $\bar{\nu}_{\mu, \tau}$ spectra $f_{\bar{\nu}_{e}}$ and $f_{\bar{\nu}_{\mu, \tau}}$ based on the simulations of ref. [3] as given in table 1 of ref. [11]. The $\bar{\nu}_{e}$ spectra have been arbitrarily normalized to one while the $\bar{\nu}_{\mu, \tau}$ spectra are normalized to 1.3 to keep the relative ratio of the number luminosities. Lower part: modified spectra $\tilde{f}_{\bar{\nu}_{e}}$ and $\tilde{f}_{\bar{\nu}_{\mu, \tau}}$, including the effects of collective neutrino oscillations, which induce a swap of the $\bar{\nu}_{e}$ and $\bar{\nu}_{\mu, \tau}$ spectra for energies above $E_{s} \approx 18 \mathrm{MeV}$ based on the normal mass hierarchy calculations of ref. [13].

These neutrons allow now the matter flow to overcome waiting point nuclei like ${ }^{64} \mathrm{Ge}$ via $(n, p)$ reactions and to continue to heavier nuclei. The associated nucleosynthesis process is called the $\nu p$-process $[10-12]$.

How far the massflow can proceed in the $\nu p$ process strongly depends on the environment conditions. In particular, the sensitivity to the temperature at neutrinodriven wind termination and the $Y_{e}$ value of the ejected matter has been investigated in ref. [14]. Here we explore the sensitivity to the luminosity and spectrum of electron antineutrinos. These are expected to be affected by collective neutrino flavor oscillations that occur in the high-neutrino-density environment surrounding the neutron star, see [15] for a recent review. Previous studies of the impact of collective neutrino oscillations in supernova nucleosynthesis have considered neutron-rich ejecta as required for $r$-process nucleosynthesis [16]. Here, we consider the case of proton-rich ejecta as predicted by recent long-term simulations $[4,5]$. Several works [13,17-19] have shown that collective neutrino oscillations swap the spectra of $\bar{\nu}_{e}$ and $\bar{\nu}_{\mu, \tau}$ neutrinos in certain energy intervals bounded by sharp spectral splits. The split energy depends on the relative fluxes of $\bar{\nu}_{e}$ and $\bar{\nu}_{\mu, \tau}$ and on the neutrino mass hierarchy $[20,21]$. In the following, as illustrated in fig. 1, we assume that collective neutrino flavor oscillations exchange the spectra of $\bar{\nu}_{e}$ and $\bar{\nu}_{\mu, \tau}$ above a certain split energy as found by Dasgupta et al. [13] in the case of normal mass hierarchy.
In general, $\bar{\nu}_{\mu, \tau}$ have a larger mean energy and a larger high-energy tail than $\bar{\nu}_{e}$. This is caused by the fact that, besides their interactions by neutral-current reactions, $\bar{\nu}_{e}$ also interact with the dense neutron star matter by charge current reactions (which is energetically not possible for supernova $\bar{\nu}_{\mu, \tau}$ antineutrinos) and hence decouple at slightly larger radii and lower temperatures. Due to the high-energy enhancement of $\bar{\nu}_{e}$, collective neutrino flavor oscillations are expected to increase the neutron production rate by antineutrino absorption on protons during the $\nu p$ process and hence its efficiency to synthesize heavy elements.

We assume that collective neutrino flavor oscillations do not change the $Y_{e}$ value which is determined by electron neutrino and antineutrino absorptions on nucleons close to the neutron star surface. Furthermore, we assume that collective neutrino oscillations occur before the onset of $\nu p$-process nucleosynthesis at distances of $\sim 500 \mathrm{~km}$. These assumptions are consistent with the recent multiangle calculations of ref. [17]. Furthemore, there are no free neutrons present and $N \sim Z$ nuclei are practically innert to neutrino absorptions. Hence, the impact of collective neutrino flavor oscillations on $\nu p$-process nucleosynthesis is to modify the rate of antineutrino absorption on protons and hence the neutron production rate.

The relevant absorption rate at distance $r$ from the neutron star center is defined as follows:

$$
\lambda_{\bar{\nu}_{e}}=\frac{1}{4 \pi r^{2}} \int_{0}^{\infty} \mathrm{d} E \sigma_{\bar{\nu}_{e}}(E) f_{\bar{\nu}_{e}}(E),
$$

with $\sigma_{\bar{\nu}_{e}}(E)$ the absorption cross-section for antineutrinos of energy $E$. For the neutrino spectra we adopt the following distribution [22]:

$$
f(\alpha, E)=\frac{L_{n}}{\Gamma(1+\alpha)}\left(\frac{1+\alpha}{\langle E\rangle}\right)^{1+\alpha} E^{\alpha} \exp \left(-\frac{(1+\alpha) E}{\langle E\rangle}\right)
$$

with the neutrino number luminosity

$$
L_{n}=\int_{0}^{\infty} \mathrm{d} E f(\alpha, E),
$$

and $\langle E\rangle$ the mean neutrino energy. The parameter $\alpha$ is fixed by the relation

$$
\left\langle E^{2}\right\rangle=\frac{\alpha+2}{\alpha+1}\langle E\rangle^{2} .
$$

It has been shown that such an $\alpha$ distribution gives a good description of the neutrino spectra calculated in supernova simulations [22].

With the assumptions above, the $\bar{\nu}_{e}$ spectrum, $\tilde{f}_{\bar{\nu}_{e}}$, modified by collective neutrino oscillations is

$$
\tilde{f}_{\bar{\nu}_{e}}(E)= \begin{cases}f_{\bar{\nu}_{e}}(E), & E<E_{s} \\ f_{\bar{\nu}_{\mu, \tau}}(E), & E>E_{s}\end{cases}
$$

According to ref. [13], we expect the split energy, $E_{s}$, to be around $18 \mathrm{MeV}$ (see fig. 1). 


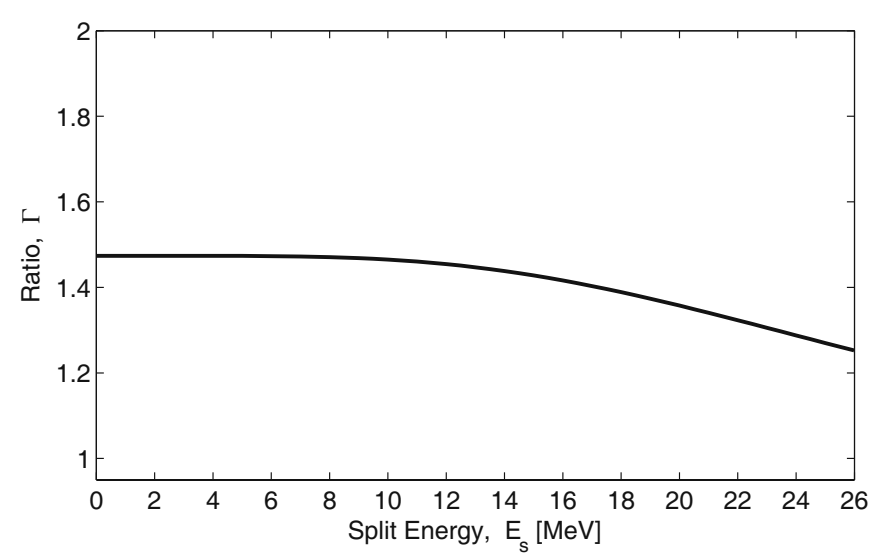

Fig. 2. Relative change $\Gamma$ of the neutron production rate with respect to the split energy $E_{s}$, calculated for the antineutrino spectra shown in the upper part of fig. 1 .

The modification of the antineutrino spectrum will affect the neutron production rate by antineutrino absorption on protons, which in turn will alter the $\nu p$-process nucleosynthesis. To quantify these effects, we define the change in the neutron production rate as

$$
\Gamma=\frac{\int_{0}^{\infty} \sigma_{\bar{\nu}_{e}}(E) \tilde{f}_{\bar{\nu}_{e}}(E) \mathrm{d} E}{\int_{0}^{\infty} \sigma_{\bar{\nu}_{e}}(E) f_{\bar{\nu}_{e}}(E) \mathrm{d} E} .
$$

To calculate the $\Gamma$ factor we approximate the antineutrino absorption cross-section by

$$
\sigma_{\bar{\nu}_{e}}(E)= \begin{cases}0, & E<\Delta, \\ 9.3 \times 10^{-44}\left(\frac{E-\Delta}{\mathrm{MeV}}\right)^{2} \mathrm{~cm}^{2}, & E>\Delta\end{cases}
$$

where $\Delta=1.293 \mathrm{MeV}$ is the neutron-proton mass difference. This approximation suffices to calculate $\Gamma$. However, for the nucleosynthesis studies we use a cross-section which also considers weak magnetism and nucleon recoil corrections [23].

Our nucleosynthesis calculations are based on the supernova simulations of a $15 \mathrm{M}_{\odot}$ star [3] and its associated nucleosynthesis $[8,11]$. In particular, luminosities and spectra parameters for all neutrino flavors are given in table 1 of ref. [11]. We have approximated these spectra by an $\alpha$ distribution, see eq. (3). For the $\bar{\nu}_{e}$ spectrum we find the parameter $\alpha_{\bar{\nu}_{e}}=2.3$ and an average neutrino energy $\left\langle E_{\bar{\nu}_{e}}\right\rangle=14.56 \mathrm{MeV}$, while for the $\bar{\nu}_{\mu, \tau}$ flavor these parameters are $\alpha_{\bar{\nu}_{\mu, \tau}}=2.3$ and $\left\langle E_{\bar{\nu}_{\mu, \tau}}\right\rangle=15.44 \mathrm{MeV}$. These spectra $f_{\bar{\nu}_{e}}(E)$ and $f_{\bar{\nu}_{\mu, \tau}}(E)$ are plotted in the upper part of fig. 1 . The lower panel shows the modified $\bar{\nu}_{e}$ spectrum including the effect of collective neutrino oscillations. We observe the increased flux of $\bar{\nu}_{e}$ neutrinos with $E>E_{s}$ in the modified spectrum. Due to the energy dependence of the neutrino absorption cross-section, see eq. (7), this increase of high-energy neutrinos will enhance the neutron production rate. This is demonstrated in fig. 2 that shows the neutron production rate to be increased by a factor $\Gamma \approx 1.4$ due to collective neutrino oscillations. Importantly, we also observe that $\Gamma$ is relatively insensitive to

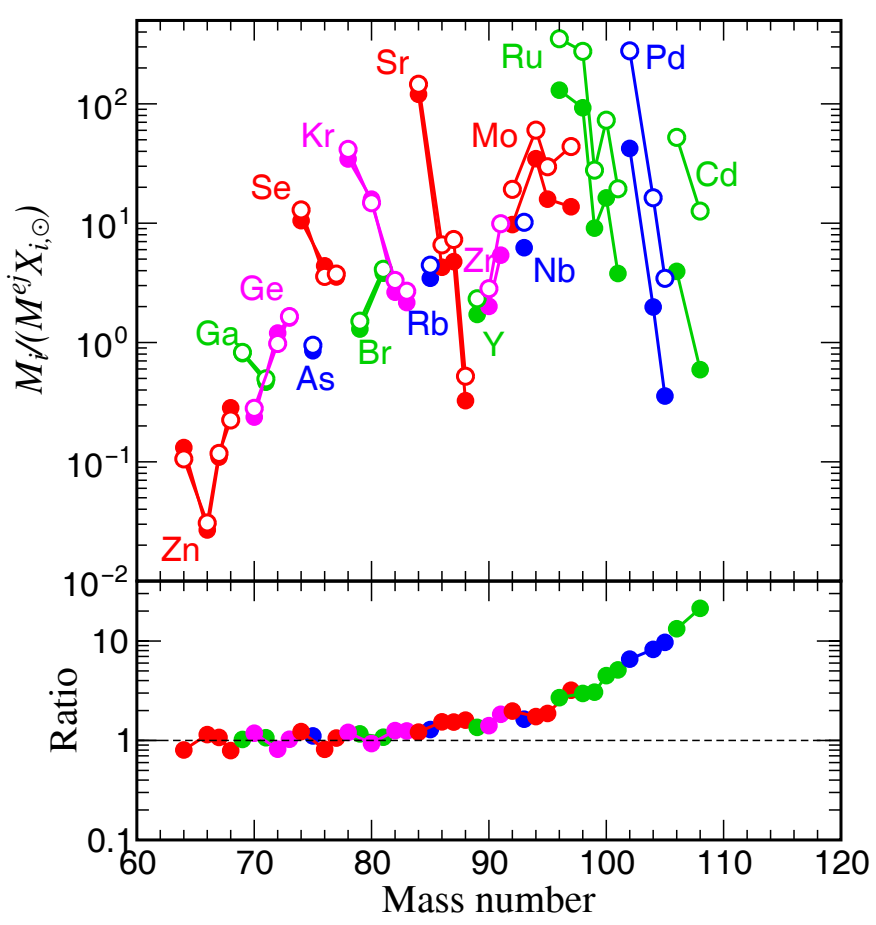

Fig. 3. Comparison of overproduction factors calculated in $\nu p$ process nucleosynthesis studies with $\Gamma=1.4$ (empty circles), hence considering collective neutrino oscillations, and $\Gamma=1.0$ (solid circles). The lower panel shows the ratio of overproduction factors for the two nucleosynthesis studies as a function of mass number.

the unknown split energy $E_{s}$, in the range up to $25 \mathrm{MeV}$. This allows us to describe the effect of collective neutrino oscillations in $\nu p$-process nucleosynthesis studies by scaling the antineutrino absorption rate by a constant factor $\Gamma$. We have considered this scaling for radii larger than $500 \mathrm{~km}$, corresponding to temperatures smaller than $3 \mathrm{GK}$ at which the $\nu p$ process operates.

Adopting $\Gamma=1.4$ from fig. 2, we have performed a nucleosynthesis calculation using a sufficiently large nuclear network considering rates for reactions mediated by the strong, electromagnetic and weak interaction and involving nuclei in the mass range up to the europium isotopes (see ref. [24] for additional details). The evolution of temperature, density and $Y_{e}$ corresponds to the trajectory labelled "1116 ms" shown in fig. 3 of ref. [8]. The abundance distributions of elements heavier than $A=64$ are compared to those obtained in a calculation, in which we choose $\Gamma=1.0$ and kept all other quantities the same. This study hence corresponds to a standard $\nu p$-process calculation without consideration of collective neutrino oscillations. In fig. 3, we show the calculated "overproduction factors" for both nucleosynthesis studies defined by the ratio $M_{i} /\left(M^{\mathrm{ej}} X_{i, \odot}\right)$, where $M_{i}$ is the produced mass of isotope $i$ and $X_{i \odot}$ is its solar-mass fraction. The total mass ejected in the supernova simulation, $M^{\mathrm{ej}}$, is taken from [8]. The enhanced neutron production due to collective neutrino oscillations has two interesting consequences. Firstly, it increases the abundances of nuclei heavier than $A=80$. In particular, the abundances of light $p$-nuclides 
$\left({ }^{92,94} \mathrm{Mo},{ }^{96,98} \mathrm{Ru}\right)$, whose production might be attributed to the $\nu p$ process, are enhanced by factors $2-3$. The increase becomes more significant for nuclides with $A>96$. This is due to the fact that the enhanced rate for neutron production increases the number of neutrons that can induce $(n, p)$ reactions on heavy nuclei. In particular, it reduces the time scale of this reaction on the $N=50$ nucleus ${ }^{96} \mathrm{Pd}$ which acts like a "seed" for the production of nuclei with $A>96$ [14]. Our results indicate that, in addition to the uncertainties in the supernova dynamics and the nuclear physics input discussed in ref. [14], collective neutrino oscillations play a very important role to determine if the $\nu p$ process can contribute to the solar inventory of light $p$-nuclei up to $A=108\left({ }^{108} \mathrm{Cd}\right)$. The production of heavier $p$-nuclei must be likely attributed to other nucleosynthesis processes.

The overproduction factors for nuclei with mass numbers $A=64,68,72$, and 76 are slightly reduced in a $\nu p$ process which considers collective neutrino oscillations. This is due to the fact that their progenitors, the $\alpha$-nuclei like ${ }^{64} \mathrm{Ge}$ or ${ }^{68} \mathrm{Se}$, serve as waiting points in the mass flow to heavier elements. As these waiting points are overcome by $(n, p)$ reactions within $\nu p$-process nucleosynthesis, the availability of a larger amount of free neutrons during the nucleosynthesis process makes the lifetimes of the $\alpha$-nuclei against $(n, p)$ reactions shorter. As a consequence, less matter is halted at the waiting points, reflected in the smaller abundances of nuclides like ${ }^{64} \mathrm{Zn}$ and ${ }^{68} \mathrm{Zn}$, which are produced via the $\alpha$-nuclei progenitors.

Recent supernova simulations $[4,5]$ show that the $\bar{\nu}_{e}$ and $\bar{\nu}_{\mu, \tau}$ spectra become increasingly similar with time after the onset of the explosion, being identical after around $10 \mathrm{~s}$ [25]. Additionally, it has been recently shown that collective neutrino flavor oscillations are suppressed during the early accretion phase of the supernova explosion [26]. Consequently, we expect a reduced impact of collective neutrino oscillations on nucleosynthesis studies both at early and late times. We also find smaller $\Gamma$ factors if we calculate the enhancement in the neutron production ratio using the neutrino spectra published for the simulations of a ONeMg core $8.8 \mathrm{M}_{\odot}$ star $[4,5]$ and a iron-core $10.8 \mathrm{M}_{\odot}$ star [5]. In both cases, $\Gamma$ is larger than 1, i.e. collective neutrino oscillations lead to increased neutron production but the obtained values, around $1 \mathrm{~s}$ after the explosion, $\Gamma=1.1\left(8.8 \mathrm{M}_{\odot}\right.$ star $)$ and $1.3\left(10.8 \mathrm{M}_{\odot}\right.$ star $)$ are smaller than discussed above. We have repeated the nucleosynthesis calculations with these $\Gamma$ factors. We observe that the overproduction factor of the elements with $A>80$ grows nearly linear with $\Gamma$. For example, the overproduction factor of ${ }^{96} \mathrm{Ru}$ is 2.3 for $\Gamma=1.3$, while it is nearly 3 for $\Gamma=1.4$.

We have studied $\nu p$-process nucleosynthesis considering the impact of collective neutrino flavor oscillations. In accordance with recent studies we assume that these result in a complete swap in the spectra of electron and non-electron flavors above a certain split energy. This swap results in enhanced electron antineutrino captures. Adopting antineutrino spectra from recent supernova simulations we find that this enhanced capture increases the amount of free neutrons, present during $\nu p$-process nu- cleosynthesis, by 20-40\%. This larger supply of neutrons boosts the matter flow to heavier nuclides and results in larger abundances of nuclides with $A>64$. Our current study makes the simplifying assumption that the collective neutrino flavor oscillations occur before the onset of $\nu p$ nucleosynthesis which allows to approximate their effect by a constant increase of the neutron production rate. Due to the intriguing effect of the oscillations on the production of light $p$-nuclides, we are planning to improve on our current study. We will perform nucleosynthesis calculations that consistently include collective neutrino flavor oscillations based on neutrino spectra and trajectories from simulations of supernova explosions.

The work has been initiated during the GSI Summer School Program. BZ thanks the Program for financial support. We thank Y.-Z. Qian and C. Volpe for useful discussions on collective neutrino oscillations. We also like to acknowledge support from HIC for FAIR, the Helmholtz Alliance EMMI and the SFB 634 at the Technical University Darmstadt. TF acknowledges support from the Swiss National Science Foundation grant number PBBSP2-133378.

Open Access This article is distributed under the terms of the Creative Commons Attribution Noncommercial License which permits any noncommercial use, distribution, and reproduction in any medium, provided the original author(s) and source are credited.

\section{References}

1. H.-T. Janka, K. Langanke, A. Marek, G. Martínez-Pinedo, B. Müller, Phys. Rep. 442, 38 (2007).

2. M. Liebendörfer, A. Mezzacappa, O.E.B. Messer, G. Martinez-Pinedo, W.R. Hix, F.-K. Thielemann, Nucl. Phys. A 719, 144 (2003).

3. R. Buras, M. Rampp, H.-T. Janka, K. Kifonidis, Astron. Astrophys. 447, 1049 (2006).

4. L. Hüdepohl, B. Müller, H. Janka, A. Marek, G.G. Raffelt, Phys. Rev. Lett. 104, 251101 (2010).

5. T. Fischer, S.C. Whitehouse, A. Mezzacappa, F.-K. Thielemann, M. Liebendörfer, Astron. Astrophys. 517, 80 (2010).

6. I.R. Seitenzahl, F.X. Timmes, A. Marin-Laflèche, E. Brown, G. Magkotsios, J. Truran, Astrophys. J. 685, 129 (2008).

7. C. Fröhlich et al., Astrophys. J. 637, 415 (2006).

8. J. Pruet, S.E. Woosley, R. Buras, H.-T. Janka, R.D. Hoffman, Astrophys. J. 623, 325 (2005).

9. X.L. Tu et al., Phys. Rev. Lett. 106, 112501 (2011).

10. C. Fröhlich et al., Phys. Rev. Lett. 96, 142502 (2006).

11. J. Pruet, R.D. Hoffman, S.E. Woosley, H.-T. Janka, R. Buras, Astrophys. J. 644, 1028 (2006).

12. S. Wanajo, Astrophys. J. 647, 1323 (2006).

13. B. Dasgupta, A. Dighe, G.G. Raffelt, A.Y. Smirnov, Phys. Rev. Lett. 103, 051105 (2009).

14. S. Wanajo, H.-T. Janka, S. Kubono, Astrophys. J. 729, 46 (2011).

15. H. Duan, G.M. Fuller, Y. Qian, Ann. Rev. Nucl. Part. Sci. 60, 569 (2010). 
16. H. Duan, A. Friedland, G.C. McLaughlin, R. Surman, J. Phys. G Nucl. Part. Phys. 38, 035201 (2011).

17. H. Duan, A. Friedland, Phys. Rev. Lett. 106, 091101 (2011).

18. S. Galais, C. Volpe, arXiv:1103.5302 [astro-ph.SR].

19. M.-R. Wu, Y.-Z. Qian, Phys. Rev. D 84, 045009 (2011).

20. G. Fogli, E. Lisi, A. Marrone, I. Tamborra, J. Cosmol. Astropart. Phys. 10, 002 (2009) arXiv:0907.5115 [hep-ph].

21. A. Mirizzi, R. Tomàs, arXiv:1012.1339 [hep-ph].
22. M.T. Keil, G.G. Raffelt, H.-T. Janka, Astrophys. J. 590, 971 (2003)

23. C.J. Horowitz, Phys. Rev. D 65, 043001 (2002).

24. A. Arcones, G. Martínez-Pinedo, Phys. Rev. C 83, 045809 (2011).

25. T. Fischer, G. Martínez-Pinedo, M. Hempel, M. Liebendörfer, in preparation (2011).

26. S. Chakraborty, T. Fischer, A. Mirizzi, N. Saviano, R. Tomàs, Phys. Rev. D 84, 025002 (2011). 